\title{
Utilização de técnicas de Arteterapia na reabilitação de pessoas com transtornos induzidos por substâncias
}

\section{Use of Art therapy techniques in the rehabilitation of people with substance-induced disorders}

DOI: $10.46919 / \operatorname{archv} 1 \mathrm{n} 6-005$

Recebimento dos originais: 01/09/2020

Aceitação para publicação: 30/10/2020

\author{
Ana Cláudia Afonso Valladares-Torres \\ Enfermeira e Arteterapeuta. Doutora em Enfermagem Psiquiátrica pela USP. Docente da Universidade de \\ Brasília (UnB) \\ Instituição: Faculdade de Ceilândia da Universidade de Brasília (FCE/UnB) \\ Endereço: Centro Metropolitano, conjunto A, lote 1 - Ceilândia Sul. Brasília, Distrito Federal - Brasil \\ E-mail: aclaudiaval@unb.br \\ Jean Carlos Marques Souza \\ Enfermeiro pela UnB. Especialista em Saúde Mental pela Fundação de Ensino e Pesquisa - Secretaria de \\ Saúde do Distrito Federal \\ Instituição: Secretaria de Saúde do Distrito Federal \\ Endereço: STN - Asa Norte, Brasília, Distrito Federal - Brasil \\ E-mail: jeancms77@gmail.com
}

\section{RESUMO}

Objetivo: identificar a percepção de um grupo de pessoas adultas com transtornos induzidos por substâncias sobre as intervenções de Arteterapia. Métodos: pesquisa qualiquantitativa, descritiva e exploratória por meio de instrumentos aplicados aos toxicômanos, como feedback individual ao final de três intervenções de Arteterapia (relaxamento, visualização criativa e atividade de arte). Resultados: os resultados das avaliações da análise de conteúdo e termômetro das emoções foram a diminuição da tensão e ansiedade, proporcionaram o relaxamento ou distração, seguida da melhora de humor, diminuição da tristeza e aumento da alegria, além de facilitarem a integração e o acolhimento grupal. Conclusão: Arteterapia é uma intervenção benéfica, que contribui significativamente para um cuidado mais humanizado no campo da saúde mental, em especial da dependência de drogas. As intervenções criativas e dinâmicas favorecem o relaxamento e, concomitantemente, diminuem a tensão e a ansiedade, bem como melhoram o humor de seus participantes.

Palavras-chave: Terapia pela arte, Saúde mental, Transtornos relacionados ao uso de substâncias, Enfermagem psiquiátrica, Serviços de saúde mental.

\begin{abstract}
Objective: to identify the perception of a group of adult people with substance-induced disorders about Art therapy interventions. Methods: qualitative, quantitative, descriptive and exploratory research using instruments applied to drug addicts, such as individual feedback at the end of three Art therapy interventions (relaxation, creative visualization and art activity). Results: the results of the content analysis and thermometer evaluations of emotions were the reduction of tension and anxiety, provided relaxation or distraction, followed by improved mood, decreased sadness and increased joy, in addition to facilitating integration and welcoming group. Conclusion: Art therapy is a beneficial intervention, which
\end{abstract}


contributes significantly to a more humanized care in the field of mental health, especially drug addiction. Creative and dynamic interventions favor relaxation and, at the same time, decrease tension and anxiety, as well as improve the mood of your participants.

Keywords: Art therapy, Mental health, Substance-Related Disorders, Psychiatric nursing, Mental health services.

\section{INTRODUÇÃO}

O consumo de substâncias psicoativas na humanidade sempre existiu na evolução históricocultural do homem, entretanto o aumento do uso abusivo e problemático dessas substâncias e suas repercussões tem sido recorrente a preocupante na atualidade, no Brasil. Representa uma das principais preocupações do Ministério da Saúde que tem enfatizado a prática de ações criativas e inovadoras dentro da saúde mental ${ }^{(1)}$.

As Arteterapias criativas e expressivas têm mostrado que podem auxiliar as pessoas a reconstruir o significado e a conexão com os outros, concentra-se nos recursos e na criatividade, o que pode favorecer aliviar os sintomas de trauma e aumentar a satisfação da vida e a esperança para o futuro ${ }^{(2)}$.

Atualmente, no Brasil, com a inclusão da Arteterapia à Política Nacional de Práticas Integrativas e Complementares pelo Ministério da Saúde ${ }^{(3)}$, as Arteterapias criativas têm ganhado destaque na mídia. Elas têm adquirido uma importância crescente na literatura, como ferramenta dentro da reabilitação em saúde em geral, em saúde mental e, em especial, com as pessoas com transtornos induzidos por substâncias.

A complexidade do tratamento e reabilitação da dependência de drogas indica a necessidade de considerar como um dos caminhos terapêuticos prioritários as Arteterapias criativas para aqueles que dificilmente verbalizam suas emoções e sentimentos e que têm dificuldade na adesão às terapias convencionais $^{(4)}$. A Arteterapia tem-se mostrado favorável na redução dos sintomas emocionais de pessoas drogadictas, pois permite que elas exponham seus sentimentos, sensações e pensamentos e, ao expor esse conteúdo, a pessoa organiza sua energia interna e estimula seu autoconhecimento ${ }^{(5)}$, características significativas para o processo de enfrentamento do transtorno. Assim, as atividades de Arteterapia podem ser um importante recurso da Enfermagem psiquiátrica em prol da recuperação dos indivíduos com dependência de drogas.

Uma revisão sistemática da literatura de 28 estudos sobre os efeitos terapêuticos da produção de arte na reabilitação psicossociais de pessoas com transtornos mentais descobriu que o uso da arte teve efeitos terapêuticos sobre o processo de reabilitação dos sujeitos. Os efeitos foram agrupados em oito categorias, construídas a partir de uma análise de conteúdo dos resultados encontrados: expressividade, 
revisão da identidade, ampliação de competências pessoais, empoderamento, reconquista da esperança, concretização de planos, reinserção social e alívio de sentimentos negativos da doença mental ${ }^{(6)}$.

A pesquisa de revisão sistemática ${ }^{(7)}$ que identificou e avaliou criticamente estudos sobre a eficácia das intervenções Arteterapias criativas sobre a dependência de drogas identificou apenas cinco pesquisas que utilizaram ensaios de controle randomizados e ensaios clínicos controlados em oito bancos de dados acadêmicos e todos eram relacionados com ao impacto positivo da musicoterapia sobre o aumento do estágio de contemplação $(0,9)$, a prontidão ao tratamento $(0,76)$, a motivação $(0,54)$ e o menor desejo de usar as substâncias psicoativas (-0,54). Entretanto não foram encontrados estudos sobre arte visual, drama, dança e terapia de movimento, ou artes na saúde. Existem poucas pesquisas sobre a eficácia e utilização de intervenções de tratamento holístico inovadores, como a terapia expressiva e Arteterapia, no campo do abuso de substâncias ${ }^{(8)}$.

A evidência da Arteterapia como uma Prática Integrativa e Complementar no enfrentamento da dependência de drogas ainda é limitada e pouco explorada. Portanto, há a necessidade de consolidar mais pesquisas científicas sobre o tema.

O objetivo geral deste estudo englobou identificar a percepção de um grupo de pessoas adultas com transtornos induzidos por substâncias sobre as intervenções de Arteterapia em um Centro de Atenção Psicossocial álcool e outras drogas do Distrito Federal. E como objetivos específicos dessa pesquisa foram estipulados: caracterizar os participantes do estudo de acordo com variáveis sociodemográficos e clínicas; e comparar o termômetro das emoções dos participantes antes e após cada intervenção de Arteterapia.

\section{MÉTODO}

A presente pesquisa teve uma abordagem qualiquantitativa, descritiva e exploratória. O estudo foi desenvolvido em um Centro de Atenção Psicossocial-álcool e outras drogas (CAPS-ad) III do Distrito Federal, que compôe a rede de saúde mental específica para pessoas adultas com problemas decorrentes ao uso abusivo de álcool e outras drogas.

O presente estudo é vinculado ao projeto da pesquisa "A Arteterapia como dispositivo terapêutico nas toxicomanias" que foi aprovado pelo Comitê de Ética em Pesquisa da Fundação de Ensino e Pesquisa em Ciências da Saúde (CEP/FEPECS), sob o CAAE n. 44625915.4.0000.5553. Todos os participantes aquiesceram a pesquisa e assinaram os Termos de Consentimento Livre e Esclarecido-TCLE.

O grupo de Arteterapia era aberto, heterogêneo quanto ao gênero e tipo de droga de dependência e formado por pessoas com idade acima de 18 anos e que tinham vínculo com a instituição, tanto pela participação nos grupos terapêuticos quanto por estarem no acolhimento integral da Unidade. O 
acolhimento integral prevê um acompanhamento diurno e noturno aos usuários do serviço como um dos recursos do Projeto Terapêutico Singular e visa à retomada, ao resgate e ao redimensionamento das relações interpessoais, ao convívio familiar e/ou comunitário com ações de manejo das situações de crise, da busca da manutenção do vínculo, do monitoramento dos projetos terapêuticos e da promoção de articulação entre os pontos de atenção com foco no cuidado e nas ações intersetoriais ${ }^{(1)}$.

As atividades foram divididas em três intervenções de Arteterapia, de uma hora e trinta minutos de duração cada uma, sempre no período vespertino. O número de participantes do grupo variou entre sete e quatorze toxicômanos adultos por intervenção e obteve-se um total de 34 participantes ao final dos três períodos de intervenção. Os objetivos gerais das intervenções de Arteterapia foram de promover o relaxamento psíquico, estimular o potencial criativo, auxiliar na reflexão pessoal e na reabilitação dos participantes. A Arteterapia foi fundamentada pela teoria da Psicologia Analítica, portanto trabalhou-se com a imaginação ativa e com o resgate de conteúdos do inconsciente para o confronto e expansão da consciência. Os símbolos expressos nas imagens contêm uma dor e uma história de cada pessoa e, ao serem expressos, favorecem o autoconhecimento ${ }^{(9)}$. Por trás de cada processo, dor e história existe uma infinidade de símbolos que permitem profundos aprendizados e que, quando ampliados, geram saltos de consciência.

As intervenções de Arteterapia foram vivências lúdicas e sensoriais e foram compostas por três modalidades de Arteterapia em cada encontro: técnicas de relaxamento, imaginação criativa e ativa e elaboração de atividade artística específica. A sala era ampla e havia colchonetes disponíveis para os participantes, bem como era composta por mesas grandes e cadeiras que possibilitaram o desenvolvimento das atividades artísticas e aparelho de som. O Quadro 1 apresenta o detalhamento das atividades de Arteterapia desenvolvidas pelos participantes durante as três intervenções.

Quadro 1 - Descrição das intervenções de Arteterapia, segundo data, números de participantes, modalidades de Arteterapia e objetivos terapêuticos. Brasília, DF, Brasil, 2020. ( $\mathrm{n}=34)$;

\begin{tabular}{|c|c|c|c|c|c|}
\hline \multirow[t]{2}{*}{$\mathbf{N}^{\mathbf{0}}$} & \multirow{2}{*}{$\begin{array}{l}\mathrm{N}^{\mathbf{o}} \mathrm{de} \\
\text { parti } \\
\text { cipan } \\
\text { tes }\end{array}$} & \multicolumn{3}{|c|}{ Modalidade de Arteterapia } & \multirow[t]{2}{*}{ Imagem/Título } \\
\hline & & Relaxamento & $\begin{array}{l}\text { Imaginação } \\
\text { criativa e ativa }\end{array}$ & Artes plásticas & \\
\hline $\mathbf{1}^{\mathrm{a}}$ & $\begin{array}{l}19, \\
13 \hat{\jmath} \\
\text { Total } \\
\text { de } 14 \\
\text { partic } \\
\text { ipante } \\
\text { s }\end{array}$ & $\begin{array}{l}\text { Alongamento } \\
\text { corporal, técnicas de } \\
\text { respiração profunda } \\
\text { e automassagem nas } \\
\text { mãos com óleo } \\
\text { essencial da arnica. }\end{array}$ & \begin{tabular}{l}
\multicolumn{3}{l}{ Visualização } \\
criativa de um \\
caminho e com sons \\
de cachoeira ao \\
fundo.
\end{tabular} & $\begin{array}{l}\text { Colagem de areia } \\
\text { sobre o papel para } \\
\text { represar a água e } \\
\text { depois joga a tinta } \\
\text { na água que se } \\
\text { expande. }\end{array}$ & $" \%$ \\
\hline $2^{a}$ & $\begin{array}{l}19, \\
12 \hat{\gamma} \\
\text { Total }\end{array}$ & $\begin{array}{l}\text { Relaxamento } \\
\text { corporal com } \\
\text { técnicas de } \\
\text { respiração profunda }\end{array}$ & $\begin{array}{l}\text { Visualização de um } \\
\text { jardim com sons de } \\
\text { pássaros ao fundo. }\end{array}$ & $\begin{array}{l}\text { Representação } \\
\text { gráfica do jardim } \\
\text { visualizado. } \\
\text { Confecção das mãos }\end{array}$ & "Floresta encantada" \\
\hline
\end{tabular}




\begin{tabular}{|c|c|c|c|c|c|}
\hline & $\begin{array}{l}\text { de } 13 \\
\text { partic } \\
\text { ipante } \\
\text { s }\end{array}$ & & & $\begin{array}{l}\text { em papel e desenho } \\
\text { e a colagem das } \\
\text { mãos no jardim. }\end{array}$ & \\
\hline $3^{a}$ & $\begin{array}{l}0 \text { Q } \\
7 \AA \\
\text { Total } \\
\text { de } 7 \\
\text { partic } \\
\text { ipante } \\
\text { s }\end{array}$ & $\begin{array}{l}\text { Movimentação } \mathrm{e} \\
\text { ativação de cada } \\
\text { parte do corpo, } \\
\text { depois técnicas de } \\
\text { relaxamento } \\
\text { corporal deitado nos } \\
\text { colchonetes e } \\
\text { respiração profunda. }\end{array}$ & $\begin{array}{l}\text { Centralização } \\
\text { entrada no estado de } \\
\text { meditação. Sons das } \\
\text { ondas do mar ao } \\
\text { fundo. }\end{array}$ & $\begin{array}{l}\text { Autorretrato pela } \\
\text { sombra e pelo } \\
\text { desenho e colagem } \\
\text { das qualidades. }\end{array}$ & facial \\
\hline
\end{tabular}

Os dados foram coletados por meio de instrumentos e aplicados aos participantes como feedback individual no final de cada uma das três intervenções de Arteterapia (relaxamento, visualização criativa e atividade de arte). Os instrumentos utilizados na coleta de dados foram:

- (A) Questionário semiestruturado elaborado pelos pesquisadores, continha os dados sociodemográficos e clínicos dos participantes, e foi desenvolvido pelos pesquisadores; objetivou indicar o perfil dos toxicômanos participantes e utilizou as variáveis como: sexo, droga de dependência, idade, tipo de vínculo com a instituição, tempo de proposta terapêutica, escolaridade, estado civil e procedência. Utilizou-se a análise quantitativa e codificaram-se os registros de dados e agregaram-se as frequências de maneira descritiva e exploratória;

- (B) Uma questão aberta, que foi elaborada pelas pesquisadoras com o objetivo de avaliar qualitativamente as intervenções de Arteterapia pelos participantes deste estudo; a questão norteadora foi: “O que significou a atividade de Arteterapia para você hoje?" foi utilizado no estudo ao final de cada intervenção e de forma individual. Utilizou-se método de análise de conteúdo $^{(10)}$, pautada em três etapas: ordenação dos dados, classificação dos dados por meio de leitura horizontal e exaustiva das falas, leitura transversal, para, finalmente, construir as categorias temáticas e análise final dos resultados da pesquisa à luz do referencial teórico;

- (C) "Termômetro das emoções", modelo adaptado ${ }^{(11)}$ que foi utilizado no início e final da cada intervenção de Arteterapia. O "Termômetro das emoções" é uma escala tipo Likert autoaplicável, cuja intensidade variava de 1 a 10; os valores mais baixos eram destinados ao polo de sentimentos negativos (como tristeza, raiva, medo/culpa, solidão, tensão/ansiedade e desamor/indiferença) e os valores mais altos o polo de sentimentos positivos (como alegria, paz interior, compreensão, integração, relaxamento e carinho). A escala de Likert é um tipo de escala de resposta psicométrica, cujas categorias de resposta servem para capturar a intensidade dos sentimentos dos participantes. O "Termômetro das emoções", ao ser medido antes e depois 
de cada intervenção, buscou avaliar a variação do nível emocional dos participantes após as intervenções de Arteterapia. O instrumento consistiu de uma tabela, com os sentimentos negativos expostos do lado esquerdo do termômetro e os positivos do lado direito. Para esse instrumento utilizou-se a análise quantitativa descritiva e exploratória.

\section{RESULTADOS}

- (A) Dados sociodemográficos e clínicos dos participantes deste estudo

A distribuição dos participantes do grupo de Arteterapia, segundo sexo, droga de dependência, idade, tipo de vínculo com a instituição, tempo de proposta terapêutica, escolaridade, estado civil, procedência e trabalho, serão desenvolvidas na Tabela 1 a seguir.

Tabela 1. Frequência das características sociodemográficas e clínicas dos participantes do grupo de Arteterapia (n=34). Brasília, DF, Brasil, 2020.

\begin{tabular}{|c|c|c|}
\hline Característica $(\mathrm{n}=34)$ & Frequência & Porcentagem \\
\hline \multicolumn{3}{|l|}{ 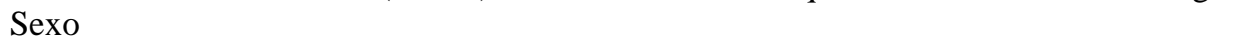 } \\
\hline Masculino & 32 & $94,1 \%$ \\
\hline Feminino & 02 & $5,9 \%$ \\
\hline \multicolumn{3}{|l|}{ Droga de Dependência } \\
\hline Álcool & 24 & $70,6 \%$ \\
\hline Crack/Cocaína & 0 & $0 \%$ \\
\hline Múltiplas Drogas & 10 & $29,4 \%$ \\
\hline \multicolumn{3}{|l|}{ Idade } \\
\hline $18-35$ & 09 & $26,5 \%$ \\
\hline $36-50$ & 18 & $52,9 \%$ \\
\hline 51 ou mais & 07 & $20,6 \%$ \\
\hline \multicolumn{3}{|l|}{ Tipo de vínculo com a instituição } \\
\hline Grupo terapêutico & 26 & $76,5 \%$ \\
\hline Acolhimento integral & 08 & $23,5 \%$ \\
\hline \multicolumn{3}{|c|}{ Tempo de proposta terapêutica no CAPS-ad } \\
\hline Menos que 1 ano & 27 & $79,4 \%$ \\
\hline Entre 1 a 4 anos & 07 & $20,6 \%$ \\
\hline Igual ou maior que 5 anos & 0 & $0 \%$ \\
\hline \multicolumn{3}{|l|}{ Grau de escolaridade } \\
\hline Nenhum & 01 & $2,9 \%$ \\
\hline Ensino fundamental & 27 & $79,4 \%$ \\
\hline Ensino médio & 06 & $17,7 \%$ \\
\hline Ensino superior & 0 & $0 \%$ \\
\hline \multicolumn{3}{|l|}{ Estado civil } \\
\hline Casado/amasiado & 11 & $32,4 \%$ \\
\hline Solteiro/divorciado/Viúvo & 23 & $67,6 \%$ \\
\hline Outros & 0 & $0 \%$ \\
\hline \multicolumn{3}{|l|}{ Procedência } \\
\hline Distrito Federal & 26 & $76,5 \%$ \\
\hline Goiás & 08 & $23,5 \%$ \\
\hline Outros & 0 & $0 \%$ \\
\hline \multicolumn{3}{|l|}{ Atividade laboral } \\
\hline Sim & 09 & $26,5 \%$ \\
\hline Não & 25 & $73,5 \%$ \\
\hline
\end{tabular}

Fonte: Banco de dados da pesquisa 
De acordo com a análise descritiva dos dados sociodemográficos e clínicos dos toxicômanos participantes, a amostra foi composta por 34 pessoas, sendo prevalente o sexo masculino com 94,1\% e observou-se que 70,6\% eram alcoolistas. A idade prevalente foi de 18 a 54 anos e com média de 36 anos, $76,5 \%$ vieram encaminhados dos grupos terapêuticos e 79,4\% tinham menos de um ano de proposta terapêutica na instituição. Do total da amostra 79,4\% tinham Ensino Fundamental completo, 67,6\% eram solteiros ou separados. A maioria dos participantes (76,5\%) era procedente do Distrito Federal e 73,5\% estavam sem desenvolver atividades laborativas no período da aplicação do quaetionário.

- (B) Avaliação das intervenções de Arteterapia pelos participantes

Após a análise do material relatado em relação à questão aberta capturaram-se seis categorias temáticas. A quantidade de respostas relatadas pelos participantes $(\mathrm{P})$ e a intervenção que foi citada $\left({ }^{\mathrm{a}}\right)$ são descritas a seguir no Quadro 2.

Quadro 2. As seis categorias de análise e seus resultados (N=34). Brasília, DF, Brasil, 2020.

Categorias
encontradas e
quantidade de
respostas

$1^{\mathrm{a}}$ - Proporcionou relaxamento ou distração - 23 respostas

le

Afirmações sobre as questões abertas ( $n^{0}$ da intervenção)

P2: Para mim foi uma terapia de relaxamento $\left(1^{\mathrm{a}}\right)$.

P3: Relaxei bastante $\left(1^{a}\right)$.

P5: Me senti bem, cheguei com raiva, mas no momento da atividade fiquei calmo, distrai e gostei da atividade $\left(1^{\mathrm{a}}\right)$.

P6: Um trabalho abstrato para desencarnar a mente de qualquer outra coisa $\left(1^{\mathrm{a}}\right)$.

P7: Significou muito, descontração $\left(1^{\mathrm{a}}\right)$.

P8: Uma terapia que trouxe paz $\left(1^{\mathrm{a}}\right)$.

P9: Ótimo. Fuga da realidade que estou vivendo $\left(1^{\mathrm{a}}\right)$.

P10: Relaxei um pouco $\left(1^{\mathrm{a}}\right)$.

P11: Boa terapia, foi um momento de descontração... me senti relaxado em uma praia $\left(1^{\mathrm{a}}\right)$.

P13: Foi relaxante $\left(1^{\mathrm{a}}\right)$.

P14: Foi relaxante $\left(1^{\mathrm{a}}\right)$.

P15: Tranquilidade $\left(2^{\mathrm{a}}\right)$.

P19: Relaxamento $\left(2^{\mathrm{a}}\right)$.

P20: Boa distração $\left(2^{\mathrm{a}}\right)$.

P21: Me distraí, esqueci os meus problemas e fiquei mais relaxado $\left(2^{\mathrm{a}}\right)$.

P22: Foi relaxante $\left(2^{\mathrm{a}}\right)$.

P23: Deu muita paz, fiquei calmo e relaxado $\left(2^{\mathrm{a}}\right)$.

P24: Fiquei calmo, ..., relaxei $\left(2^{\mathrm{a}}\right)$.

P26: Significou paz $\left(2^{\mathrm{a}}\right)$.

P28: A vivência foi divertida $\left(3^{\mathrm{a}}\right)$.

P31: Se distraiu e ficou calmo $\left(3^{\mathrm{a}}\right)$.

P32: Foi uma forma de preencher o tempo $\left(3^{\mathrm{a}}\right)$.

$\mathrm{P} 34:$ A vivência me distraiu com suas atividades $\left(3^{\mathrm{a}}\right)$.

$2^{\mathrm{a}}-$ Gostou da atividade e/ou gerou alegria - 13
P1: Gostei bastante da atividade de hoje $\left(1^{\mathrm{a}}\right)$.

P4: Foi muito agradável $\left(1^{\mathrm{a}}\right)$.

P11: Foi legal... alegria em estar aqui $\left(1^{\mathrm{a}}\right)$. 


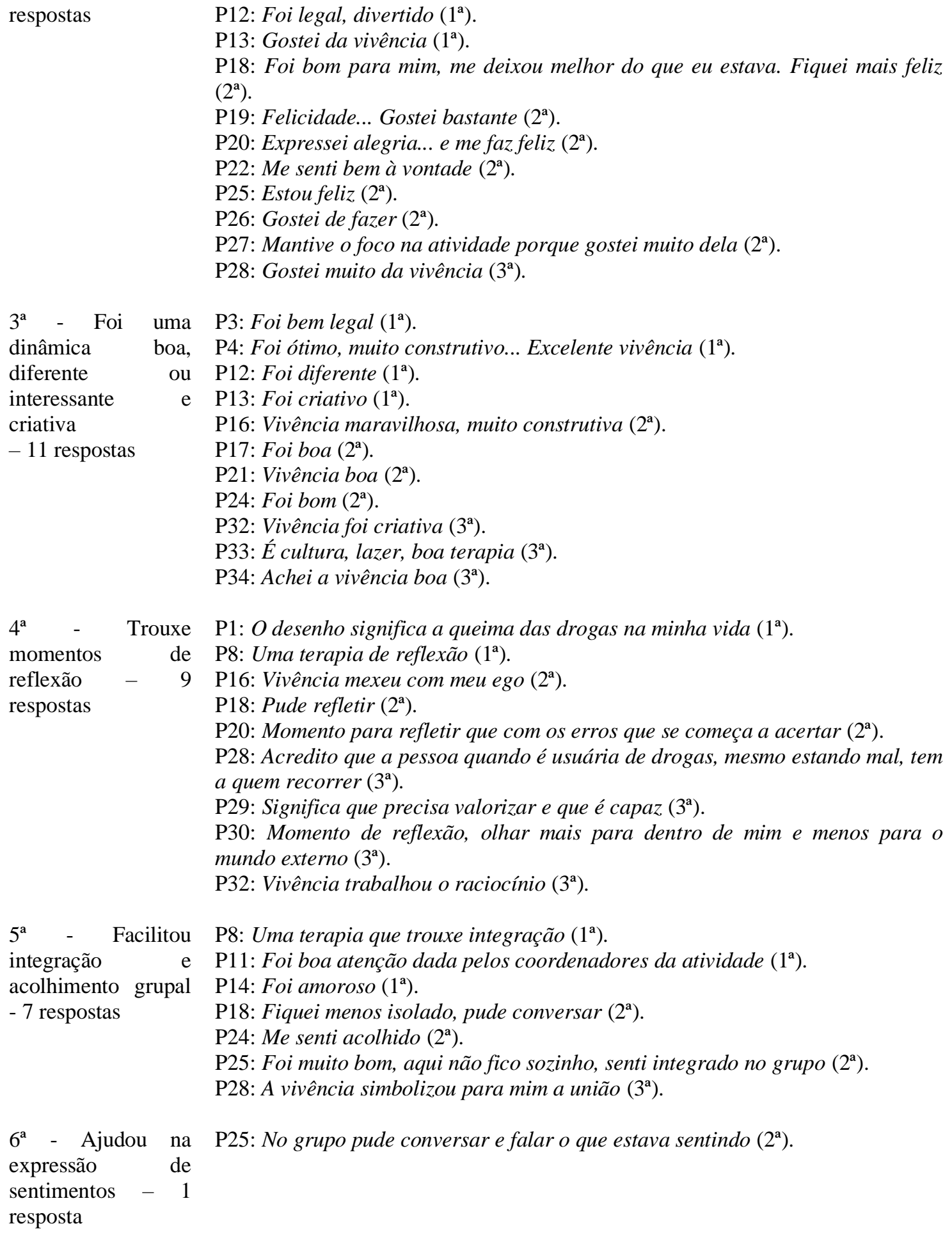

Trinta e quatro participantes responderam ao questionário durante três intervenções de Arteterapia e foram apreendidas seis categorias temáticas, a mais prevalente foi "proporcionou relaxamento ou distração" com 23 respostas, seguida da categoria "gostou da atividade e/ou gerou alegria" com treze respostas. A resposta "foi uma dinâmica boa, diferente ou interessante e criativa" obteve onze respostas, a categoria "trouxe momentos de reflexão" atingiu nove respostas, já a categoria "facilitou integração e 
acolhimento grupal" alcançou sete respostas e, finalmente, a categoria “ajudou na expressão de sentimentos" alcançou apenas uma resposta.

\section{- (C) Avaliação das emoções antes e após cada intervenção de Arteterapia pelos participantes}

Quando à frequência da intensidade dos sentimentos pelos participantes referentes ao questionado “Termômetro das emoções" (11) - comparativa antes e após as intervenções de "Arteterapias criativas" são apresentadas no Quadro 3.

Quadro 3: Resultado da alteração da intensidade dos sentimentos referente ao questionado "Termômetro das emoções" e comparativa após as intervenções de "Arteterapias criativas". (n=35). Brasília, DF, 2020.

\begin{tabular}{|c|c|c|c|c|c|}
\hline Sentimento & $\begin{array}{c}1^{\circ} \text { intervenção } \\
N=14\end{array}$ & $\begin{array}{c}2^{\mathrm{a}} \\
\text { intervenção } \\
\mathrm{N}=13\end{array}$ & $\begin{array}{c}3^{\mathbf{a}} \\
\text { intervenção } \\
\mathrm{N}=7\end{array}$ & $\begin{array}{c}\text { TOTAL } \\
\mathbf{N}=34\end{array}$ & $\begin{array}{l}\text { Total por } \\
\text { paciente }\end{array}$ \\
\hline Tristeza-Alegria & $\uparrow 30$ pontos & $\uparrow 17$ pontos & $\uparrow 9$ pontos & $\uparrow 56$ pontos & $\begin{array}{c}\uparrow \mathbf{1 , 6} \\
\text { ponto/paciente }\end{array}$ \\
\hline $\begin{array}{c}\text { Raiva-Paz } \\
\text { interior }\end{array}$ & $\uparrow 14$ pontos & $\uparrow 22$ pontos & $\uparrow 6$ pontos & $\uparrow 42$ pontos & $\begin{array}{c}\uparrow \mathbf{1 , 2} \\
\text { ponto/paciente }\end{array}$ \\
\hline $\begin{array}{l}\text { Medo/Culpa- } \\
\text { Compreensão }\end{array}$ & $\uparrow 9$ pontos & $\uparrow 21$ pontos & $\uparrow 16$ pontos & $\uparrow 46$ pontos & $\begin{array}{c}\uparrow 1,4 \\
\text { ponto/paciente }\end{array}$ \\
\hline $\begin{array}{c}\text { Solidão- } \\
\text { Integração }\end{array}$ & $\uparrow 16$ pontos & $\uparrow 24$ pontos & $\uparrow 10$ pontos & $\uparrow 50$ pontos & $\begin{array}{c}\uparrow \mathbf{1 , 5} \\
\text { ponto/paciente }\end{array}$ \\
\hline $\begin{array}{l}\text { Tensão/Ansiedad } \\
\text { e-Relaxamento }\end{array}$ & $\uparrow 42$ pontos & $\uparrow 43$ pontos & $\uparrow 13$ pontos & $\uparrow 98$ pontos & $\begin{array}{c}\uparrow \mathbf{2 , 9} \\
\text { pontos/paciente }\end{array}$ \\
\hline $\begin{array}{c}\text { Desamor/Indifere } \\
\text { nça-Carinho }\end{array}$ & $\uparrow 8$ pontos & $\uparrow 22$ pontos & $\uparrow 6$ pontos & $\uparrow 36$ pontos & $\begin{array}{c}\uparrow \mathbf{1 , 1} \\
\text { ponto/paciente }\end{array}$ \\
\hline Total progresso & $\uparrow 119$ pontos & $\uparrow 149$ pontos & $\uparrow 60$ pontos & $\begin{array}{c}\uparrow 328 \\
\text { pontos }\end{array}$ & $\begin{array}{c}\uparrow 628 \text { pontos/ } \\
\uparrow \mathbf{9 , 7} \\
\text { pontos/paciente }\end{array}$ \\
\hline $\begin{array}{l}\text { Média total por } \\
\text { paciente }\end{array}$ & $\begin{array}{c}\uparrow 8,5 \\
\text { pontos/pacient } \\
\mathrm{e}\end{array}$ & $\begin{array}{c}\uparrow 11,5 \\
\text { pontos/pacient } \\
\mathrm{e}\end{array}$ & $\begin{array}{c}\uparrow 8,5 \\
\text { pontos/pacient } \\
\mathrm{e}\end{array}$ & $\begin{array}{c}\uparrow 9,7 \\
\text { pontos/pac } \\
\text { iente }\end{array}$ & $\begin{array}{c}\uparrow \mathbf{1 , 6} \\
\text { ponto/paciente/ } \\
\text { sentimento }\end{array}$ \\
\hline
\end{tabular}

Legenda: $\uparrow$ Aumento de pontos

Fonte: Banco de dados da pesquisa

A análise geral e descritiva dos sentimentos dos toxicômanos participantes mostra que os sentimentos negativos foram diminuídos, ao passo que todos os positivos obtiveram progresso. Os sentimentos que mais obtiveram progresso foram relacionados à diminuição da tensão e ansiedade e ao aumento do relaxamento, simultaneamente, atingiu a uma pontuação total de 98 pontos e equivale-se a 2,9 ponto/participante. Seguido da diminuição da tristeza e aumento da alegria com 56 pontos (1,6 ponto/participante), diminuição da solidão e aumento da integração atingiu 50 pontos (1,5 ponto/participante) e, ainda, diminuição do medo e culpa e aumento da compreensão com 46 pontos (1,4 ponto/participante). Também houve, entretanto com menos intensidade a diminuição da raiva e aumento da paz com 42 pontos (1,2 ponto/participante) e, finalmente, diminuição do desamor e indiferença pelo 
aumento do carinho, também simultaneamente, com ganho de 36 pontos, correspondeu a 1,1 ponto/participante.

\section{DISCUSSÃO}

- (A) Dados sociodemográficos e clínicos dos participantes deste estudo

Em relação ao predomínio do sexo masculino $(94,1 \%)$ desta pesquisa, esses dados corroboram com os achados de outros estudos em CAPSad que tiveram uma pontuação de 76\%, 82\%, 84,0\% e 92\% conforme autores que estudaram o perfil sociodemográfico de usuários de CAPS-ad do Brasil (12-15). Pode-se verificar que sobre o tipo de droga de dependência houve predominância do álcool em 70,6\% dos participantes, sobretudo muitos deles apesar de eleger o álcool como substância de dependência, também consumiam outras drogas psicoativas. Dados que são semelhantes aos encontrados em outros perfis sociodemográficos de usuários de CAPS-ad pelo Brasil. Em outros estudos, a incidência foi de 29\% ${ }^{(12)}$, e $26,0 \%{ }^{(13)}$. Isoladamente, o álcool é a droga mais consumida pelos dependentes de drogas usuários dos CAPS-ad, entretanto os usuários fazem geralmente uso de várias substâncias em geral ${ }^{(14)}$.

A amostra se apresentou, predominantemente, de adultos-jovem, a faixa etária variou de 18 a 54 anos e em média de 36 anos. Tais dados são semelhantes aos encontrados na maioria dos estudos no Brasil: os entrevistados prevaleceram entre 20 a 40 anos (57\%) em um estudo ${ }^{(12)}$, enquanto entre 18 a 41 anos $(68 \%)$ em outro estudo ${ }^{(13)}$. Possuem idade igual ou superior a 35 anos $(41,9 \%)^{(14)}$, e entre 18 e 65 anos, com a média de $41 \operatorname{anos}^{(15)}$.

Houve a prevalência de participantes de solteiros ou separados $(67,6 \%)$. A predominância de solteiros neste estudo é confirmada por outros estudos brasileiros: encontrou-se $52,5 \%{ }^{(12)}, 61 \%{ }^{(13)}$, $61,5 \%^{(14)}$, e $46 \%{ }^{(15)}$ de solteiros.

Quanto à escolaridade, foi predominante o Ensino Fundamental incompleto em 79,4\%. Dados que também se assemelham aos de outras pesquisas brasileiras: Gonçalves e Nunes (2014), Santos, Carvalho e Miranda (2014), Mastroianni, Macris, Gomes e Camargo (2016) e Oliveira et al. (2017) que também tiveram predominância deste nível de escolaridade apresentou índices de $61 \%^{(12)}, 51 \%{ }^{(13)}, 41,7 \%^{(14)} \mathrm{e}$ $58,9 \%{ }^{(15)}$.

A maioria dos participantes apresentou não estar em situação empregatícia ativa em 73,5\%. Bem como nas outras pesquisas, houve predomínio de desempregados em 51,5\% ${ }^{(12)} \mathrm{em} 60 \%{ }^{(13)} \mathrm{e}$ em $45,4 \%{ }^{(15)}$. Destoante de somente $25,3 \%{ }^{(14)}$ de desempregados em que a maioria estava vinculada a alguma atividade produtiva, seja ela acadêmica ou laborativa. A pesquisa atual encontrou um percentil mais alto que as demais pesquisas, provavelmente porque foi considerado a ausência de trabalho ativo no momento e não 
apenas estar desempregado e foram incluídas nesta categoria também, as pessoas que se encontravam de licença, aposentadas entre outras.

A maioria dos participantes $(76,5 \%)$ veio encaminhada dos grupos terapêuticos e poucos do acolhimento integral, realçou-se que 79,4\% tinham menos de um ano de proposta terapêutica na instituição. (76,5\%) da amostra eram procedentes do Distrito Federal, o mesmo ocorreu em outra pesquisa brasileira $^{(13)}$ no qual $81 \%$ dos entrevistados eram residentes da região de localização do CAPS-ad.

Por questões sociais, culturais e diferentes papéis sociais estabelecidos na sociedade, os homens são mais propensos, em sua maioria, a usar drogas psicoativas do que as mulheres. O álcool é uma droga lícita e bastante consumida socialmente e mais bem aceita na cultura brasileira, o que pode facilitar a compra e ingestão da substância por um número maior de pessoas e consequentemente favorecer a dependência ${ }^{(1)}$.

A dependência de drogas é um fenômeno completo e desencadeia uma série de repercussões negativas em várias dimensões do sujeito envolvido, sejam sociais, psicoafetivas, emocionais, culturais, físicas, culturais, espirituais, profissionais e financeiras, escolaridade, como vistos nos levantamentos sociodemográficos e clínicos das pessoas com transtornos induzidos por substâncias ${ }^{(1)}$. As substâncias psicoativas representam frequentemente um alívio imediato às angústias e aos medos existenciais ou representa uma inclusão social e, ao mesmo tempo, estigma pessoal e social ${ }^{(1)}$. A dependência de drogas pode provocar baixa autoestima, baixa produtividade no trabalho, prejuízos escolares e familiares, além do aumento da morbidade e da mortalidade, comportamento de risco e o envolvimento a situações mais frequentes de violência ${ }^{(1)}$. A baixa escolaridade entre os dependentes de drogas pode estar associada à baixa qualificação, o que pode gerar desemprego a agravar o problema da dependência e levar à vulnerabilidade social e à criminalidade ${ }^{(13)}$.

- (B) Avaliação das intervenções de Arteterapia pelos participantes e

- (C) Avaliação das emoções antes e após cada intervenção de Arteterapia pelos participantes

Os resultados das avaliações da análise de conteúdo e a do termômetro das emoções seguiram-se semelhantes em relação às respostas mais prevalentes, como a diminuição da tensão e ansiedade e o proporcionou relaxamento ou distração que obtiveram uma pontuação maior, seguida da melhora de humor, isto é, diminuição da tristeza e aumento da alegria. Registrou-se também que as intervenções de Arteterapia facilitaram a integração e o acolhimento grupal.

Ao avaliar o impacto da Arteterapia grupal, utilizou-se a técnica de marmorização, sobre os níveis de ansiedade de pacientes com esquizofrenia e transtorno bipolar em serviços psiquiátricos, verificou-se que houve diminuição significativa nos sintomas negativos (embotamento afetivo, apatia, anedonia, falta 
de sociabilidade ou déficit de atenção) e positivos (delírios, alucinações, fala desorganizada, comportamento bizarro ou afeto inadequado) nos pacientes com esquizofrenia e em pacientes de ambos diagnósticos a Arteterapia contribuiu para diminuir significativamente os níveis de ansiedade ${ }^{(16)}$.

Os benefícios terapêuticos encontrados por Valladares-Torres e Moreira (2020) do uso do “Termômetro das Emoções” em grupo de mulheres dependentes de drogas antes e após às intervenções de Arteterapia revelou que o processo ajudou a promover o relaxamento, favoreceu a integração e melhorou o estado de ânimo ao longo as intervenções de Arteterapia $^{(17)}$.

Terapias complementares de mente-corpo que incluíram várias técnicas, entre elas o relaxamento e a meditação, o uso da imaginação ativa e a Arteterapias, foram eficazes em diminuir os efeitos colaterais comuns relacionados ao câncer, incluíram-se náuseas e vômitos, dor, fadiga, ansiedade, sintomas depressivos, melhoria da qualidade de vida geral e na função imune e dos hormônios do estresse ${ }^{(18)}$.

Ouvir músicas relaxantes pode ser uma técnica terapêutica efetiva e complementar nas intervenções terapêuticas para o tratamento da toxicodependência, pois pode diminuir a compulsão pela droga e/ou melhorar a estimulação cognitiva na dependência de drogas ${ }^{(19)}$.

Várias pesquisas têm ressaltado o potencial das Arteterapias criativas para minimizar sintomas depressivos e ansiosos ${ }^{(20-23)}$. Um ensaio randomizado, longitudinal e controlado sobre avaliação de um programa de Arteterapia com pessoas com esquizofrenia aprissionados revelou diminuição da ansiedade, depressão, raiva e sintomas psiquiátricos negativos (embotamento afetivo, apatia, anedonia, falta de sociabilidade ou déficit de atenção) e mostrou melhoria na socialização com o grupo de pares ${ }^{(20)}$.

Um estudo randomizado controlado sobre os efeitos da Arteterapia com argila em adultos com transtorno depressivo maior, em tratamento ambulatorial, indicou um efeito significativo em sinais depressivos, saúde geral e bem-estar corpo-mente-espírito (todos p <0,05). Assim, o estudo concluiu que a Arteterapia pode auxiliar a regulação emocional e beneficiar vários aspectos da saúde mental em adultos depressivos $^{(21)}$.

Um estudo, ao avaliar a eficácia da Arteterapia com base na apreciação de pinturas famosas sobre a angústia de pacientes com câncer que receberam radioterapia, foi concentrado, em particular, na ansiedade, depressão e sintomas relacionados ao câncer medidos em três momentos distintos, pré, durante e no pós sessões de Arteterapia e utilizaram-se as escalas de Ansiedade e Depressão Hospitalar (HADS), de Depressão de Hamilton (HDRS) e de Avaliação de Sintomas de Edmonton (ESAS). Os pesquisadores concluíram que houve melhoria significativa nos níveis de ansiedade e de depressão relacionadas ao câncer e, igualmente, reduziu a prevalência de ansiedade e depressão severas durante o tratamento do câncer $^{(22)}$. 
Um estudo comparativo da Arteterapia, ao utilizar as técnicas de pintura em aguarela, em pacientes com câncer que recebem quimioterapia mostrou melhoria significativa na qualidade de vida global $(F=7,87, P=0,001)$ e na pontuação de depressão $(F=7,80, P=0,001)$ dos participantes ${ }^{(23)}$. Outro estudo, ao envolver sessões de Arteterapia aplicadas a pacientes com câncer submetidos a tratamento quimioterápico, revelou que a Arteterapia melhorou o humor, a ansiedade e a dor entre todos os pacientes. Os benefícios foram evocados imediatamente após a sessão e persistiram pelo menos 48-72 horas em todos os participantes ${ }^{(24)}$.

A Arteterapia como uma possibilidade de envolver as pessoas com dependência de drogas e outros transtornos tem sido utilizada como uma prática em saúde mental, além da promoção de bem-estar e auxílio na estabilização da doença e na autonomia do sujeito ${ }^{(25)}$. Outra pesquisa desenvolvida com adolescentes dependentes de drogas destaca o uso terapêutico do estêncil grafite nas oficinas de artes do CAPS-ad favoreceu a vinculação e aceitação do tratamento, melhorou da autoestima e valorização pessoal dos participantes, assim como trouxe uma coloração e singularização do espaço de fala e escuta dos jovens vinculados ao tratamento ${ }^{(26)}$.

Estudos descritivos e qualitativos de Arteterapia tanto pela modalidade do desenho quanto da modelagem com dependentes de drogas adultos ressaltaram que o processo arteterapêutico foi eficaz na organização psíquica dos participantes, facilitou a conscientização de símbolos ocultos ou reconciliou problemas emocionais e permitiu as trocas sociais com o grupo de pares, respectivamente. Acrescentaram-se em ambos os estudos que a Arteterapia auxilia no autoconhecimento e na reabilitação dos toxicômanos participantes ${ }^{(27-34)}$.

Artigo de reflexão sobre as experiências artístico-culturais na área da Saúde Mental no modelo da Reforma Psiquiátrica no Brasil revelou que as pessoas em sofrimento mental se tornam atores de suas vidas, capazes de produzir novas possibilidades de expressão e facilita a inclusão social ${ }^{(35)}$.

Destaca-se a importância da arte como instrumento terapêutico, que, por meio das diferentes formas de expressão e de criação, favorece o resgate da espontaneidade, a ampliação da consciência e maior autointegração das pessoas. Aspectos que contribuem para que a pessoa encontre novas formas de se perceber e ao mundo, e, simultaneamente, de criar e recriar como indivíduo, de ajustar criativamente suas vivências e de encontrar sua própria forma de ser e de se expressar, e, de modo igual, possibilita um crescimento saudável da sua saúde mental ${ }^{(36)}$.

A formação de grupos terapêuticos inovadores pode obter melhorias psicossociais em usuários em sofrimento psíquico grave, como criatividade, comunicação, autoestima, habilidades sociais, bem como serve de motivação profissional, sem necessitar de recursos financeiros dispendiosos ${ }^{(37) \text {. }}$ 
As artes terapias criativas e expressivas têm mostrado que podem auxiliar as pessoas a reconstruir o significado e a conexão com os outros, concentra-se nos recursos e na criatividade, o que pode favorecer aliviar os sintomas de trauma e aumentar a satisfação da vida e a esperança para o futuro ${ }^{(2)}$.

\section{CONSIDERAÇÕES FINAIS}

Após as avaliações da análise de conteúdo sobre a verbalização dos participantes acerda da percepção das intervenções de Arteterapia e dos resultados do termômetro das emoções pré e pós sessões identificou-se uma diminuição da tensão e da ansiedade, proporcionaram o relaxamento ou distração, além da melhoria de humor, com a diminuição da tristeza e do aumento da alegria e da integração grupal.

Dessa forma, a Arteterapia é uma intervenção benéfica, significativa e que contribui significativamente para um cuidado mais humanizado no campo da saúde mental, em especial da dependência de drogas. As intervenções de forma criativa e dinâmica favorecem o relaxamento e, concomitantemente, diminuem a tensão e a ansiedade, bem como melhoram o humor de seus participantes. O enfermeiro em saúde mental pode incorporar recursos inovadores e criativos por meio das Arteterapia na sua prática diária, o que ampliaria o leque de experiências e experimentações, poderia facilitar o vínculo terapêutico e maior adesão dos participantes.

Sugere-se a realização de novas pesquisas sobre Arteterapia no âmbito da dependência de drogas sejam feitas para poder suprir a carência de pesquisas na área. Como limitações desta pesquisa destacamse o curto prazo e o número limitado de participantes.

\section{REFERÊNCIAS}

1. SNPD - Secretaria Nacional de Políticas sobre Drogas. Efeitos de substâncias psicoativas. 11. ed. Brasília: SENADE, 2017. Módulo 2.

2. DeMott MAM, Jakobsen M, Wentzel-Larsen T, He T. A controlled early group intervention study for unaccompanied minors: can expressive arts alleviate symptoms of trauma and enhance life satisfaction? Scandinavian Journal of Psychology. 2017; 58(6):510-8. Doi: 10.1111/sjop.12395.

3. Brasil, Ministério da Saúde. Portaria n. 849, de 27 de março de 2017: Inclui a Arteterapia, Ayurveda, Biodança, Dança Circular, Meditação, Musicoterapia, Naturopatia, Osteopatia, Quiropraxia, Reflexoterapia, Reiki, Shantala, Terapia Comunitária Integrativa e Yoga à Política Nacional de Práticas Integrativas e Complementares. Diário Oficial da União, 28 Mar 2017, n. 60. Seção 1, pág. 68. Disponível:

http://www.lex.com.br/legis_27357131_PORTARIA_N_849_DE_27_DE_MARCO_DE_2017.aspx. [capturado em 03 abril 2017]. 
4. Valladares-Torres ACA. Arteterapias criativas com mulher dependente de múltiplas drogas - um estudo de caso. Revista Científica de Arteterapia Cores da Vida. 2018; 25(1):26-37. Disponível em: https://www.abcaarteterapia.com/revista-cores-da-vida.

5. Valladares-Torres ACA, Rodrigues LTA. Eficácia de programa de Arteterapia com grupo de mulheres com dependência de drogas. Revista de Arteterapia Proceso Creativo Transformación. 2020; (7):50-6. Disponible en: www.arteterapiarevista.com.ar

6. Correia PR, Torrente MO. N. Efeitos terapêuticos da produção artística para a reabilitação psicossocial de pessoas com transtornos mentais: uma revisão sistemática da literatura. Cad. saúde colet. 2016; 24(4):487-95.

7. Megranahan K, Lynskey MT. Do creative arts therapies reduce substance misuse? A systematic review. The Arts in Psychotherapy. 2018; 57:50-8.

8. Adedoyin C, Burns NM, Jackson HM, Franklin S. Revisiting holistic interventions in substance abuse treatment. Journal of Human Behavior in the Social Environment. 2014; 24(5):538-46.

9. Jung CG. Chegando ao inconsciente. In: Jung CG. O homem e seus símbolos. Nova ed. Rio de Janeiro: Harper Collins, 2016. Cap.1. p.18-103.

10. Bardin L. Análise de conteúdo. Lisboa, Portugal: Edições 70 - Brasil: edição revista e ampliada; 2011.

11. Vergara ER. Arteterapia y liminalidad: una intervención de Arteterapia en una paciente con cáncer terminal [monografia]. Santiago de Chile (Chile): Facultad de Artes, Escuela de Pos-graduação em Terapias de Arte, Mención Arteterapia; 2010.

12. Gonçalves TS, Nunes, S. Perfil dos usuários do Centro de Atenção Psicossocial Álcool e Drogas CAPS AD. Perquirere. 2014; 11(2):169-78.

13. Santos RCA, Carvalho SR, Miranda FAN. Perfil socioeconômico e epidemiológico dos usuários do Centro de Atenção Psicossocial Álcool e Drogas II de Parnamirim, RN, Brasil. Rev. Bras. Pesq. Saúde. 2014; 16(1):105-11.

14. Mastroianni FC, Macris CE, Gomes JR, Camargo PJ. Perfil sociodemográfico de um CAPSad e sua funcionalidade segundo os usuários. Rev. Psicol. Saúde. 2016; 8(2):3-16.

15. Oliveira VC, Capistrano FC, Ferreira ACZ, Kalinke LP, Felix JVC, Maftum MA. Perfil sociodemográfico e clínico de pessoas atendidas em um CAPS ad do sul do Brasil. Rev baiana enferm. 2017; 31(1):e16350.

16. Akhan LU, Atasoy N. Impact of marbling art therapy activities on the anxiety levels of psychiatric patients. Journal of Human Sciences. 2017; 14(2):2121-8.

17. Valladares-Torres ACA, Moreira DSS. Alterações das emoções nas intervenções de Arteterapia com o uso de histórias aplicadas a mulheres dependentes de drogas. Revista Científica de Arteterapia Cores da Vida. 2020; 27(1):18-28. Disponível em: https://www.abcaarteterapia.com/revista-cores-da-vida 
18. Carlson LE, Zelinski E, Toivonen K, Flynn M, Qureshi M, Piedalue K-A, Grant R. Mind-body therapies in cancer: what is the latest evidence? Current Oncology Reports. 2017; 19(10):67.

19. Stamou V, Chatzoudi T, Stamou L, Romo L, Graziani P. Music-assisted systematic desensitization for the reduction of craving in response to drug-conditioned cues: a pilot study. The Arts in Psychotherapy. 2016; 51:36-45.

20. Qiu HZ, Ye ZJ, Liang MZ, Huang YQ, Liu W, Lu ZD. Effect of an art brut therapy program called go beyond the schizophrenia (GBTS) on prison inmates with schizophrenia in mainland China-A randomized, longitudinal, and controlled trial. Clin Psychol Psychother. 2017; 24(5):1069-78. Doi: 10.1002/cpp.2069

21. Nan JKM, Ho RTH. Effects of clay art therapy on adults outpatients with major depressive disorder: a randomized controlled trial. J Affect Disord. 2017; 1(217):237-45.

22. Lee J, Choi MY, Kim YB, Sun J, Park EJ, Kim JH, Kang M, Koom WS. Art therapy based on appreciation of famous paintings and its effect on distress among cancer patients. Qual Life Res. 2017; 26(3):707-15.

23. Bozcuk H, Ozcan K, Erdogan C, Mutlu H, Demir M, Coskun S. A comparative study of art therapy in cancer patients receiving chemotherapy and improvement in quality of life by watercolor painting. Complement Ther Med. 2017;30:67-72.

24. Doll M, Roshon SG, Stone ER, Butler RS. Evaluation of art therapy on mood, anxiety, and pain levels in patients with cancer undergoing chemotherapy treatment. Journal of Clinical Oncology. 2017; 35(15 suppl):e21713.

25. Pendleton PJ. Painting a path of well-being: Art therapy as a link to mental health treatment. Art Therapy. 2013; 16(1):31-6.

26. Machado AC. O uso terapêutico do estêncil grafite com adolescentes na oficina de artes do CAPS-ad Cascavel. Revista Educação, Artes e Inclusão. 2013; 7(1):41-57.

27. Valladares-Torres ACA, Oliveira AL, Lago DMSK, Souza JM. O uso do desenho projetivo da autoimagem no tratamento de mulheres toxicômanas vítimas de violência - uma experiência em Arteterapia. Rev. Arteterapia da AATESP. 2018; 9(1):4-30. Disponível em: file:///G:/Arteterapia/Gravação-

CD/Revista\%20AATESP/Revista\%20Arteterapia\%20AATESP\%20v9\%20n1\%202018.pdf.

28. Fernades MC, Valladares-Torres ACA, O desenho como terapia e elucidação de sonhos com usuários de um Centro de Atenção Psicossocial-álcool e outras drogas do Distrito Federal. Rev Científica Arteterapia Cores da Vida. 2019; 26(2):17-24. Disponível em: https://www.abcaarteterapia.com/revistacores-da-vida

29. Angelim SMAV, Valladares-Torres ACA. O desenho 'metáfora da chuva' como instrumento de comunicação terapêutica da problemática drogadição. Rev Científica Arteterapia Cores da Vida. 2019; 26(1):48-57. Disponível em: https://www.abcaarteterapia.com/revista-cores-da-vida

30. Valladares-Torres ACA, Santiago ECL. Arteterapia com grupo de mulheres em sofrimento mental relacionado ao uso abusivo de substâncias psicoativas. In: Barbosa AFC (Org.). Ciências da Saúde: uma 
abordagem pluralista. Piracanjuba, GO: Conhecimento Livre, 2020. Cap.12. p.122-147. Vol.1. Ebook. Doi: 10.37423/200802403 Disponível em: https://conhecimentolivre.org/ebook/ciencias-da-saude-umaabordagem-pluralista/

31. Soares ALS, Valladares-Torres ACA. Percepção de um grupo de mulheres toxicômanas em Arteterapia sobre o Centro de Atenção Psicossocial. Rev Científica Arteterapia Cores da Vida. 2020; 27(1):29-40. Disponível em: https://www.abcaarteterapia.com/revista-cores-da-vida

32. Valladares-Torres ACA. Arteterapia no cuidado à saúde mental de mulheres adictas no acolhimento integral. In: Freitas SAA (Org.). Coletânea Saúde e Bem-Estar: teorias e práticas. São Luís: Pascal, 2020. Cap.3. p.34-52. Vol.2. Doi: 10.29327/523958. Disponível em: https://editorapascal.com.br/wpcontent/uploads/2020/10/SA\%C3\%9ADE-E-BEM-ESTAR-VOL.-02.pdf

33. Torres ACAV, Lima VHR. Desenhos que revelam o processo de adoecimento de mulheres usuárias de um CAPS-ad III. Archives of Health. 2020; 1(5):364-386. Doi: https://doi.org/10.46919/archv1n5-020 Disponível em: https://latinamericanpublicacoes.com.br/ojs/index.php/ah/article/view/96

34. Valladares-Torres ACA, Anicárcio NS, Lago DMSK, Araújo AHIM. A Ponte da Vida: evolução psicossocial de homem dependente de drogas a partir de representações gráficas. Rev Científica Arteterapia Cores da Vida. 2019; 26(2):3-16. Disponível em: https://www.abcaarteterapia.com/revistacores-da-vida

35. Amarante P, Torres EHG. Madness and cultural diversity: innovation and rupture in experiences of art and culture from Psychiatric Reform and the field of Mental Health in Brazil. Interface (Botucatu). 2017; 21(63):763-74.

36. Barreira MML, Martins KBGS. A arte como instrumento terapêutico sob o enfoque da gestalt-terapia. Rev. FSA. 2017; 14(3):193-208.

37. Torre AM, Muros IA, Carracedo MVG, Jiménez FJD, Aceituno PG. Taller de cine y recursos audiovisuales para la recuperación en salud mental. Revista Española de Enfermería de Salud Mental. 2017; (2):28-32. Disponible en: http://www3.uah.es/revenfermeriasaludmental/index.php/REESMAEESME/article/viewFile/3/12 